\title{
Electromagnetic Characterization of the CFRPs Anisotropic Conductivity: Modeling and Measurements
}

\author{
Hocine Menana and Mouloud Féliachi \\ Université de Nantes, IREENA-IUT, BP 406, 37 Bd de l'université, 44602 Saint Nazaire, France. \\ E-mails: hocine.menana@univ-nantes.fr , mouloud.feliachi@univ-nantes.fr \\ Tel : + $33(0) 240172636$, Fax : + $33(0) 240172618$
}

PACS. 82.20.Wt Computational modeling; simulation - 81.70.Ex Nondestructive testing: electromagnetic testing, eddy-current testing

Shortened title: Electromagnetic characterization of CFRPs

\begin{abstract}
This work deals with the characterization of the conductivity tensor of a carbon fiber reinforced polymer composite (CFRP) thin plate. We propose a contactless method based on the eddy current non destructive testing technique. The used eddy current sensor consists of a ferrite torus on which a winding is wound. The torus is of a rectangular section and contains a thin air-gap in which the thin CFRP plate is inserted. We developed analytical relations giving the longitudinal and transversal conductivities of the CFRP plate as functions of the impedances variations of the eddy current sensor, corresponding to the orientations of the carbon fibers parallel and transverse to the direction of the torus width which is much greater than its thickness. The analytical relations are developed by inverting interpolation functions of curves giving the variations of the sensor impedances as functions of the longitudinal and transversal conductivities of the CFRP plate. These curves are obtained by a numerical model based on a simplified integro-differential formulation in terms of the electric vector potential in the CFRP plate, coupled to the magnetic circuit equations in the ferrite torus. The modeling results are supported by measurements.
\end{abstract}

\section{Introduction}

Carbon fiber reinforced composites (CFRPs) present superior characteristics compared to metals; they are both robust and light weight which make them widely used especially in aerospace applications.

Knowing the electrical conductivity of CFRPs, as well as the other electromagnetic properties, is of great importance for various applications such as electromagnetic compatibility [1], eddy current non-destructive testing [2], induction heating [3], and for some electromechanical applications [4]. Unfortunately, these proprieties are not known in advance; they are determined either by using the homogenization techniques [3], or by appropriate measurements once the material is constructed [5]-[6].

The four points bridge technique has been used to determine the electrical conductivity of CFRPs [5]; however, the high anisotropy and low dimensionality of these materials make this technique in many cases difficult and unreliable [6]. Contactless methods, based on resonant circuits [6] and on the physics of the eddy currents [7], have been proposed to determine the global electrical conductivity of CFRPs; however, the anisotropy has been weakly treated.

In this work, an inverse model based on the physics of eddy current is developed to determine the conductivity tensor of a CFRP thin plate. The used eddy current sensor consists of a ferrite torus on which a winding is wound. The torus is of a rectangular section and contains a thin air-gap in which the thin CFRP plate is inserted. The use of the ferrite torus allows us to eliminate the perturbations due to the lift-off variations, and to impose a normal magnetic source field in the thin CFRP plate.

\section{The experimental device and method principle}

A schematic view of the experimental device is described in Figure 1. A CFRP ply of thickness $\delta$ and of unknown conductivity is positioned in the air-gap $(e)$ of a ferrite torus of a rectangular section and on which a winding is wound. The torus winding is connected to a high precision impedance analyzer. The inner and outer radii of the torus are denoted respectively by $R_{i}$ and $R_{e}$. The width $(\tau)$ of the torus is much greater than its thickness $\left(R_{e}-R_{i}\right)$. The numerical values of the system parameters are given in Table 1.

A CFRP ply is of about $125 \mu \mathrm{m}$ thickness. It is constituted of carbon fibers of about $10 \mu \mathrm{m}$ diameter embedded in an electrically non conductive matrix. The conductivities vary between $5 \times 10^{3}$ and $5 \times 10^{4} \mathrm{~S} / \mathrm{m}$ in the direction of the fibers, and between 10 and $10^{2} \mathrm{~S} / \mathrm{m}$ in the transverse direction, depending on the type and the volume fraction of 
the fibers [8]. The conductivities in the directions parallel and transverse to fibers are denoted respectively by $\left(\sigma_{L}\right)$ and $\left(\sigma_{T}\right)$.

The insertion of the CFRP ply in the torus air-gap leads to a variation of the inductor impedance due to induced currents in the ply. As the torus is of a rectangular section and the fibers are unidirectional in the ply, this impedance variation depends on the orientation $(\theta)$ of the CFRP ply (i.e., carbon fibers) in the torus air-gap.

According to the physics of the eddy currents, the longitudinal or transversal conductivity of the CFRP ply can not act alone on the impedance variation of the inductor; the cancellation of one of them implies the cancellation of the eddy currents. We propose thus to express the variations of the inductor resistance as functions of the summation of product of the longitudinal and transversal conductivities weighted by coefficients to be determined.

$$
\left|\Delta R_{\theta}\right|=\sum_{i} a_{\theta}^{i} \sigma_{L}^{\alpha_{\theta}^{i}} \sigma_{T}^{\beta_{\theta}^{i}}
$$

Notice that the relation (1) applies to any inductor that realizes a distinction between the directions parallel and transverse to fibers. Limited to one term, and for the orientations $\left(\theta=0^{\circ}\right.$ and $\left.\theta=90^{\circ}\right)$, the relation (1) becomes

$$
\begin{aligned}
& \left|\Delta R_{0}\right| \approx a_{0} \sigma_{L}^{\alpha_{0}} \sigma_{T}^{\beta_{0}}, \\
& \left|\Delta R_{90}\right| \approx a_{90} \sigma_{L}^{\alpha_{90}} \sigma_{T}^{\beta_{90}} .
\end{aligned}
$$

In (1-a) and (1-b) $\Delta \mathrm{R}_{0}$ and $\Delta \mathrm{R}_{90}$ are the resistance variations of the inductor corresponding respectively to the positions where the fibers are oriented in the direction of the torus width and in the transverse direction.

The relations (1-a) and (1-b) are inverted to express the conductivities $\sigma_{L}$ and $\sigma_{T}$ of the CFRP plate as functions of $\Delta \mathrm{R}_{0}$ and $\Delta \mathrm{R}_{90}$; after some manipulations, we obtain the following relations

$$
\begin{gathered}
\sigma_{L}=\left[\left(\frac{\left|\Delta R_{0}\right|}{a_{0}}\right)\left(\frac{\left|\Delta R_{90}\right|}{a_{90}}\right)^{-1}\right]^{\frac{\beta_{90}}{\left(\alpha_{0} \beta_{90}-\alpha_{90} \beta_{0}\right)}} \\
\sigma_{T}=\left(\frac{\left|\Delta R_{90}\right|}{a_{90}}\right)^{\frac{1}{\beta_{90}}}\left[\left(\frac{\left|\Delta R_{0}\right|}{a_{0}}\right)^{-\alpha_{90}}\left(\frac{\left|\Delta R_{90}\right|}{a_{90}}\right)^{\frac{\alpha_{90} \beta_{0}}{\beta_{90}}}\right]^{\frac{1}{\left(\alpha_{0} \beta_{90}-\alpha_{90} \beta_{0}\right)}}
\end{gathered}
$$

The parameters $a_{0}, a_{90}, \alpha_{0}, \alpha_{90}, \beta_{0}$ and $\beta_{90}$ are determined from (1-a) and (1-b), by using curves giving the variations of $\left|\Delta \mathrm{R}_{0}\right|$ and $\left|\Delta \mathrm{R}_{90}\right|$ as functions of $\sigma_{L}$ and $\sigma_{T}$ which are constructed by numerical simulation (figure 4$)$. At this end, the relations (1-a) and (1-b) are linearized as follows

$$
\begin{array}{r}
\alpha_{0} \ln \left(\sigma_{L}\right)+\beta_{0} \ln \left(\sigma_{T}\right)+\ln \left(a_{0}\right) \approx \ln \left|\Delta R_{0}\right|, \\
\alpha_{90} \ln \left(\sigma_{L}\right)+\beta_{90} \ln \left(\sigma_{T}\right)+\ln \left(a_{90}\right) \approx \ln \left|\Delta R_{90}\right| .
\end{array}
$$

The parameters $a_{0}, \alpha_{0}$ and $\beta_{0}$, as well as $a_{90}, \alpha_{90}$ and $\beta_{90}$, are determined by a simple identification on three points; i.e. solving 1-c and 1-d for three chosen values of $\left|\Delta \mathrm{R}_{0}\right|$ and $\left|\Delta \mathrm{R}_{90}\right|$ taken from figure 4.

\section{The electromagnetic modeling}

The evaluation of the inductor resistance variation passes through the determination of the eddy currents in the CFRP ply which is done numerically. The numerical modeling of the eddy current in the CFRP ply is coupled to the magnetic circuit equations of the inductor (torus + winding) to avoid integrating the latter in the numerical 
modeling. The modeled system is represented schematically in the figure 2 . The influence of the CFRP ply is represented by a fictitious loop carrying a current $I$ representing the eddy currents in the CFRP ply. The fictitious current $I$ is calculated numerically.

We assume that the magnetic flux density $B$ is homogeneously distributed in the torus section; thus it can be expressed as follows

$$
\tilde{B}=\frac{\mu_{0} \mu_{r}}{\pi\left(R_{e}+R_{i}\right)+\left(\mu_{r}-1\right) e}\left\{N \tilde{I}_{s}-\tilde{I}(\tilde{B})\right\}
$$

In (4), $\mu_{0}$ and $\mu_{r}$ are respectively the free space permeability and the relative permeability of the ferrite torus; $N$ is the number of turns of the torus winding; $R_{i}$ and $R_{e}$ are respectively the inner and outer radii of the torus; $e$ is the air-gap thickness; $\tilde{I}_{s}$ is the source current and $\tilde{I}$ is the current in the fictitious loop, representing the eddy current in the CFRP ply which in turn depends on the flux density. The tilde sign means that the values are complex.

The electromagnetic problem in the CFRP ply is formulated by an integro-differential equation involving the electric vector potential [9]. The frequencies used are such that the CFRP ply thickness is much smaller than the skin depth corresponding to its maximum conductivity; in addition, the flux density is normal to its plane. The thin shell approximation is thus applied to the formulation which is reduced to involve only the normal component of the electric vector potential.

Considering the Cartesian coordinates where the CFRP ply thickness is oriented in the $z$ axis direction, the electromagnetic problem in the CFRP ply is formulated as follows

$$
\left\{-\left(\begin{array}{ll}
\partial_{y} & -\partial_{x}
\end{array}\right)\left(\begin{array}{ll}
\rho_{x x}(\theta) & \rho_{x y}(\theta) \\
\rho_{y x}(\theta) & \rho_{y y}(\theta)
\end{array}\right)\left(\begin{array}{c}
\partial_{y} \\
-\partial_{x}
\end{array}\right)\right\} \tilde{T}_{z}(r)+\frac{i \omega \mu_{0} \delta}{4 \pi} \int_{S_{p l y}} \frac{\partial_{y^{\prime}} \tilde{T}_{z}\left(r^{\prime}\right)\left(x-x^{\prime}\right)+\partial_{x^{\prime}} \tilde{T}_{z}\left(r^{\prime}\right)\left(y-y^{\prime}\right)}{\left|\vec{r}-\vec{r}^{\prime}\right|^{3}} d s=-i \omega \tilde{B}(r)
$$

In (5), $S_{p l y}$ denote the CFRP ply surface and $\delta$ is its thickness, $\omega$ is the angular frequency, $\partial_{\mathrm{u}}$ denote the derivative with respect to the coordinate $u(u=x, y), r$ and $r$ ' denote points identified respectively by the coordinates $(x, y)$ and $\left(x^{\prime}, y^{\prime}\right)$ and $|\vec{r}-\vec{r}|$ is the distance between them; $T_{z}$ represents the $z$ component of the electric vector potential, $i$ is the imaginary unit $\left(i^{2}=-1\right)$ and $\rho_{u v}(\theta)(u=x, y ; v=x, y)$ is a component of the resistivity tensor of the CFRP plate depending on the orientation $(\theta)$ of the fibers in the $X Y$ plane.

The components of the eddy current density in the CFRP ply are calculated as follows

$$
\left(\begin{array}{l}
\tilde{J}_{x} \\
\tilde{J}_{y}
\end{array}\right)=\left(\begin{array}{c}
\partial y \tilde{T}_{z} \\
-\partial x \tilde{T}_{z}
\end{array}\right)
$$

The current in the fictitious loop, representing the effect of the eddy currents in the CFRP ply of length $L$, width W and thickness $\delta$, is calculated as follows (see figure 3)

$$
\tilde{I}=\delta \int_{0}^{W / 2} \tilde{J}_{x} d y(\text { at } x=L / 2)=\delta \int_{0}^{L / 2} \tilde{J}_{y} d x(\text { at } y=W / 2)
$$

The equality between the two integrals in (7), evaluated respectively at the positions $x=L / 2$ and $y=W / 2$, is due to the total current conservation in the plate.

The equations (4) to (7) are to be solved iteratively. We start by evaluating (4) considering only the source current $\tilde{I}_{s}$ and then solve (5), evaluate (6) and (7) and then reevaluate (4) by introducing the updated value of the current $\tilde{I}$. The iteration process is repeated until convergence; the convergence test can be realized either on the magnetic flux density or on the electric vector potential.

Once the eddy currents are determined in the CFRP ply, the variation of the inductor resistance is evaluated by the following relation, where $\tilde{J}_{u(u=x, y)}^{*}$ denotes the conjugate of $\widetilde{J}_{u(u=x, y)}$. 


$$
\Delta R(\theta)=\frac{\delta}{I_{s}^{2}} \int_{S_{p l y}}\left[\tilde{J}_{x}^{*}\left(\rho_{x x}(\theta) \tilde{J}_{x}+\rho_{x y}(\theta) \tilde{J}_{y}\right)+\tilde{J}_{y}^{*}\left(\rho_{y x}(\theta) \tilde{J}_{x}+\rho_{y y}(\theta) \tilde{J}_{y}\right)\right] d s
$$

\section{Results and validation}

The system is simulated at a frequency of $100 \mathrm{KHz}$. The differential and integral terms of (5) are discretized by using respectively the finite difference method and the moment method. The resulting algebraic system is solved by using the bi-conjugate gradient method.

Figure 3 represents zooms on the eddy currents repartitions in the CFRP ply corresponding to the orientations of the fibers in the directions parallel $(\theta=0)$ and transverse $(\theta=\pi / 2)$ to the width $\tau$ of the torus. The influence of the anisotropy on the eddy currents repartition in the CFRP ply is noticeable; the eddy currents are stretched in the direction of the fibers (axis of the high conductivity).

Figure 4 represents the variations of $\left|\Delta \mathrm{R}_{0}\right|$ and $\left|\Delta \mathrm{R}_{90}\right|$ as functions of $\sigma_{L}$ and $\sigma_{T}$ in the intervals corresponding to the standard conductivities of CFRPs. We can remark that both $\left|\Delta \mathrm{R}_{0}\right|$ and $\left|\Delta \mathrm{R}_{90}\right|$ vary in a significant way with the variation of the transverse conductivity $\sigma_{T}$; however, for a fixed $\sigma_{T}$, when $\sigma_{L}>\sigma_{T}$, the variation of $\sigma_{L}$ leads to weak variations of $\left|\Delta \mathrm{R}_{0}\right|$ and $\left|\Delta \mathrm{R}_{90}\right|$. These results are used to identify the parameter $a_{0}, a_{90}, \alpha_{0}, \alpha_{90}, \beta_{0}$ and $\beta_{90}$ by using (1-c) and (1-d).

The resistance variations $\left|\Delta \mathrm{R}_{0}\right|$ and $\left|\Delta \mathrm{R}_{90}\right|$ by have been measured by means of the impedance analyzer at a frequency of $100 \mathrm{KHz}$, and then the conductivities $\sigma_{L}$ and $\sigma_{T}$ have been identified by using the relation (2) and (3). As expected, the weak variations of $\left|\Delta \mathrm{R}_{0}\right|$ and $\left|\Delta \mathrm{R}_{90}\right|$ according to $\sigma_{L}$ lead to a degenerate identification of the latter by means of (2). The conductivity $\sigma_{L}$ is thus determined by (1-a) once $\sigma_{T}$ is determined by means of (3). The obtained results are given in Table 2 .

Figure 5 represents the measured variation of the inductor impedance $(R+i X)$ according to frequency, without and with the CFRP ply in the torus air-gap, with the fibers oriented in the directions parallel and transverse to the torus width. We remark that the presence of the CFRP ply and the orientation of its fibers in the torus air-gap lead to a significant variation of the resonance frequency and a significant variation of the inductor resistance at these resonance frequencies. These parameters constitute, thus, interesting features for anisotropic material characterization.

To check the validity of the identified conductivities, we compared between the calculated and measured values of the resistance variation of an air-cored coil above the characterized CFRP ply for different frequencies. A schematic view of the validation device is given in Figure 6. The coil characteristics are given in Table 1, and the comparison results are presented in Figure 7.

The measured and calculated results of the air-cored coil resistance variation are globally in agreement. When looking to the low frequencies $(f<700 \mathrm{KHz})$, the CFRP ply conductivities seems to be overestimated since the calculated resistance variations is greater than the measured ones. The situation is inversed at high frequencies where the measured resistance variations become greater than the calculated ones; this is due to capacitive couplings between fibers in the CFRP ply which are not taken into account in the modeling.

\section{Conclusion}

We proposed a contactless method for the characterization of the anisotropic conductivity of a thin CFRP plate. A simplified modeling is proposed and measurement results are provided. The modeling needs to be improved by taking into account the capacitive coupling in the CFRPs. We have seen that the high anisotropy of CFRPs leads to a weak dependence of the impedance variation of the inductor on the highest conductivity, which results in errors in the determination of the latter.

Measurements showed that the resonance frequency of the inductor, as well as the value of its resistance at this resonance frequency, vary in a significant way with the variation of the orientation of the CFRP ply fibers in the torus air-gap. This phenomenon constitutes an interesting alternative for characterizing anisotropic materials.

\section{Acknowledgments}

Work supported by Nantes Métropole. 


\section{References}

1. Igor Maria De Rosa, Riccardo Mancinelli, Fabrizio Sarasini, Maria Sabrina Sarto, and Alessio Tamburrano, IEEE Trans. Magn. 51, 700 (2009)

2. Gerhard Mook, Rolf Lange, Ole Koeser, Composites Science and Technology 61, 865 (2001)

3. D. Trichet, E. Chauveau,and Javad Fouladgar, IEEE Trans. Magn. 36, 1193 (2000)

4. S. B. Pratap, and W. F. Weldon, IEEE Trans. Magn. 32, 437 (1996)

5. J. B. Park, T. K. Hwang, H. G. Kim and Y. D. Doh, Smart Mater. Struct. 16, 57 (2007)

6. C. Zeller, A. Denenstein, and G. M. T. Foley, Review of Scientific Instruments 50, 602 (1978)

7. Isabelle Dufour and Dominique Placko, J. Phys. III France 3, 1065 (1993)

8. R. Grimberg, A. Savin, R. Steigmann, A. Bruma, in Proceedings of the $8^{\text {th }}$ International Conference of the Slovenian Society for Non-Destructive Testing, Portorož, 2005, p. 223

9. H. Menana, M. Féliachi, IEEE Trans. Magn. 45, 1008 (2009)

\section{Figures and tables captions}

Fig. 1. Schematic view of the experimental device

Fig. 2. Equivalent electric circuit of the experimental device

Fig. 3. Zooms on the eddy currents repartition in the CFRP ply for the orientations of the fibers parallel $(\theta=0)$ and transverse $(\theta=\pi / 2)$ to the torus width $(\tau)$

Fig. 4. Variations of $\left|\Delta \mathrm{R}_{0}\right|$ and $\left|\Delta \mathrm{R}_{90}\right|$ as function of $\sigma_{L}$ and $\sigma_{T}$

Fig. 5. The measured variation of the inductor impedance as function of the frequency, without the CFRP ply and with the CFRP ply where the fibers are oriented in the directions parallel $(\theta=0)$ and transverse $(\theta=\pi / 2)$ to the torus width $(\tau)$

Fig. 6. Schematic view of the validation device

Fig. 7. Comparison between calculation and measurement results of the resistance variation of the air-cored coil above the characterized CFRP ply

Table 1. The numerical values of the system parameters

Table 2. Measurement and identification results 
Figures

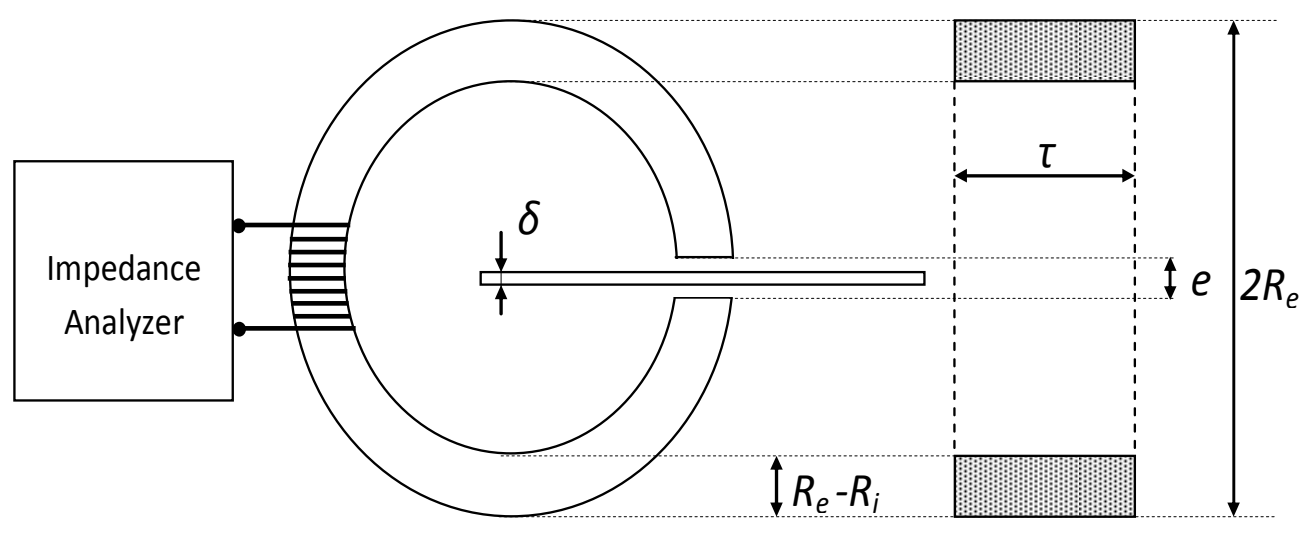

Fig. 1

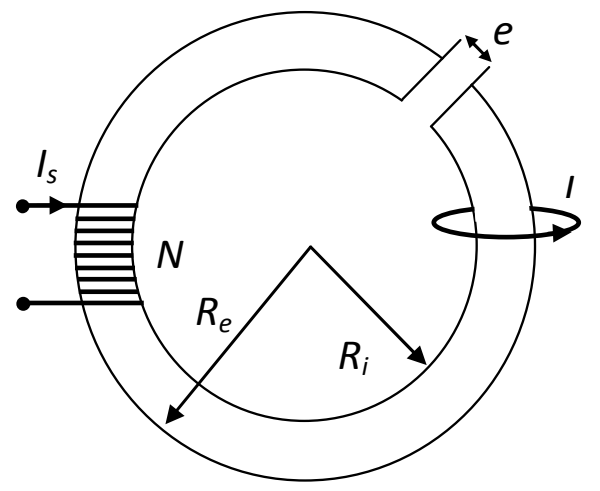

Fig. 2 
$\theta=0$
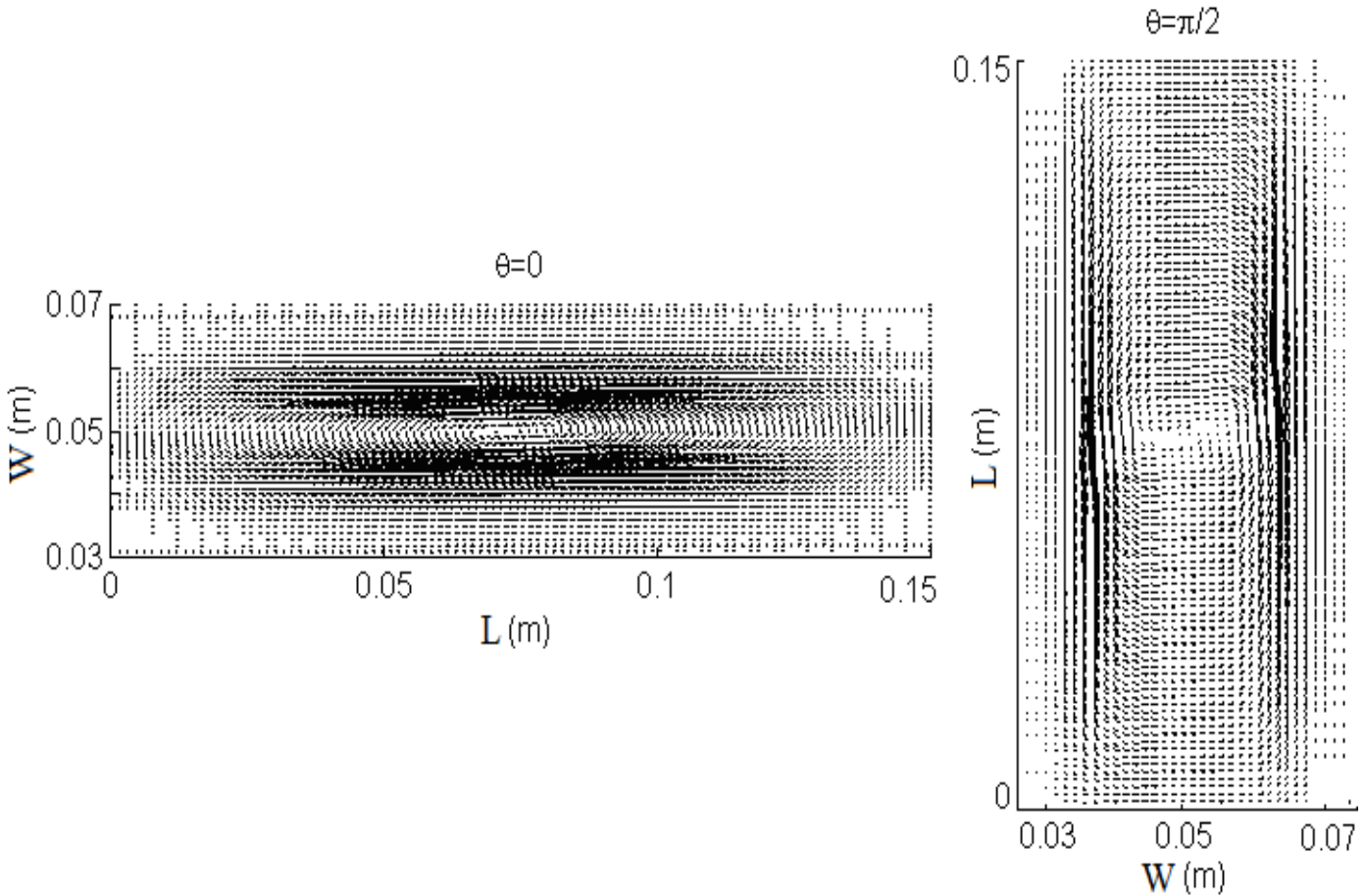

Fig. 3

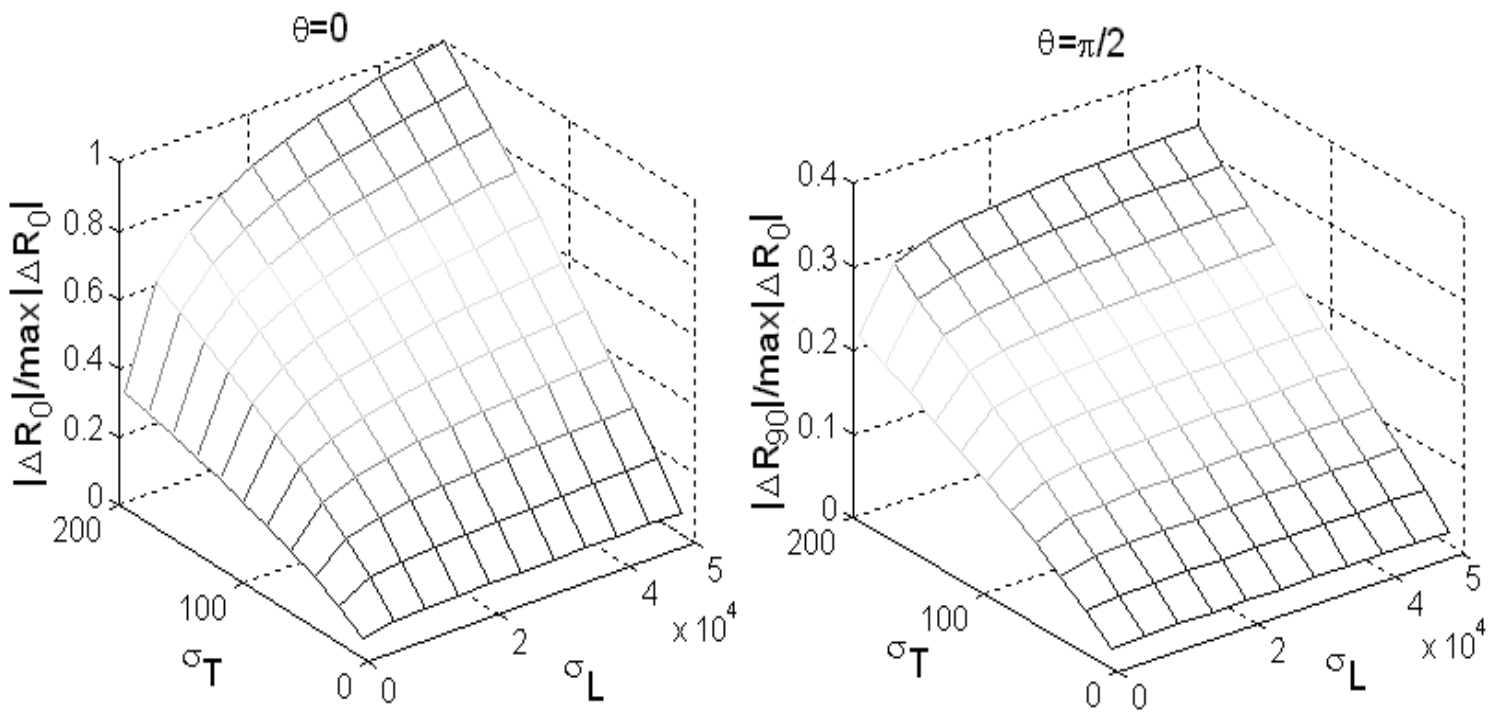

Fig. 4 

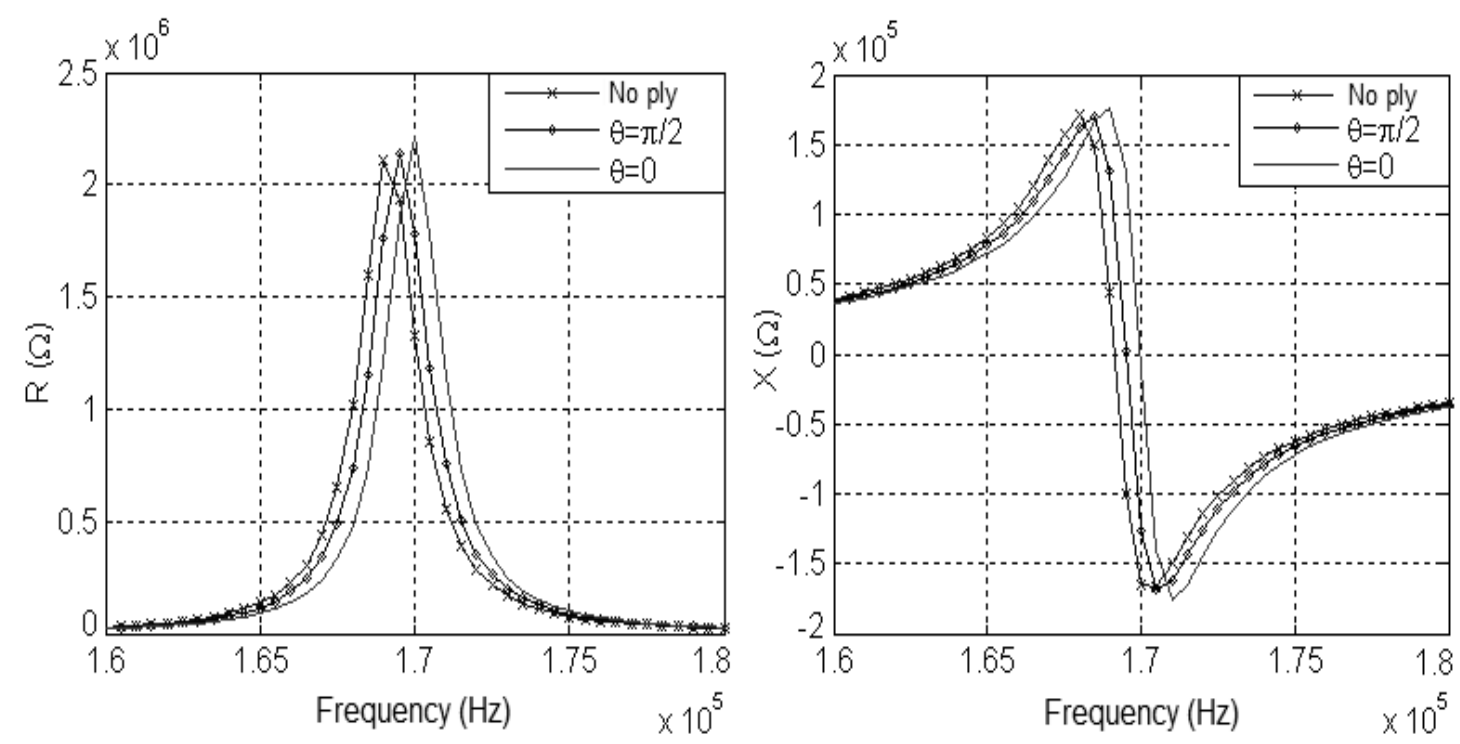

Fig. 5

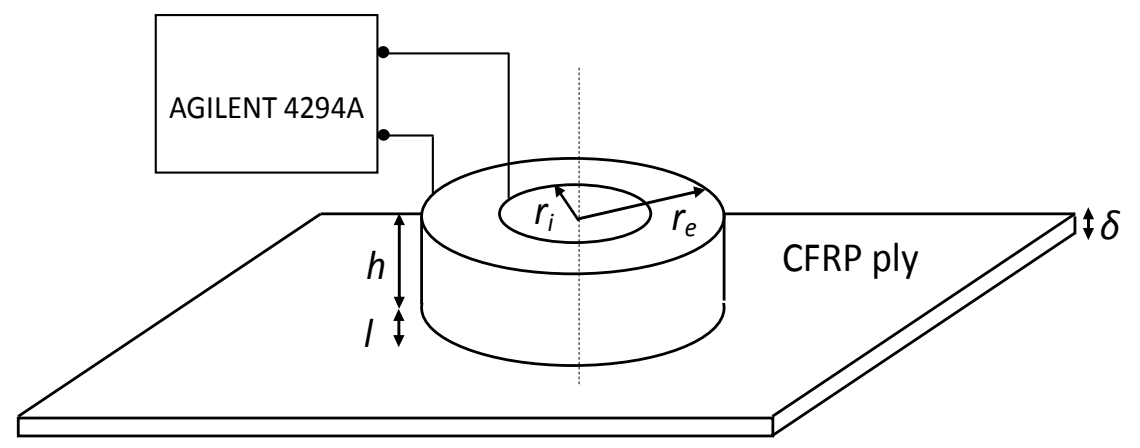

Fig. 6

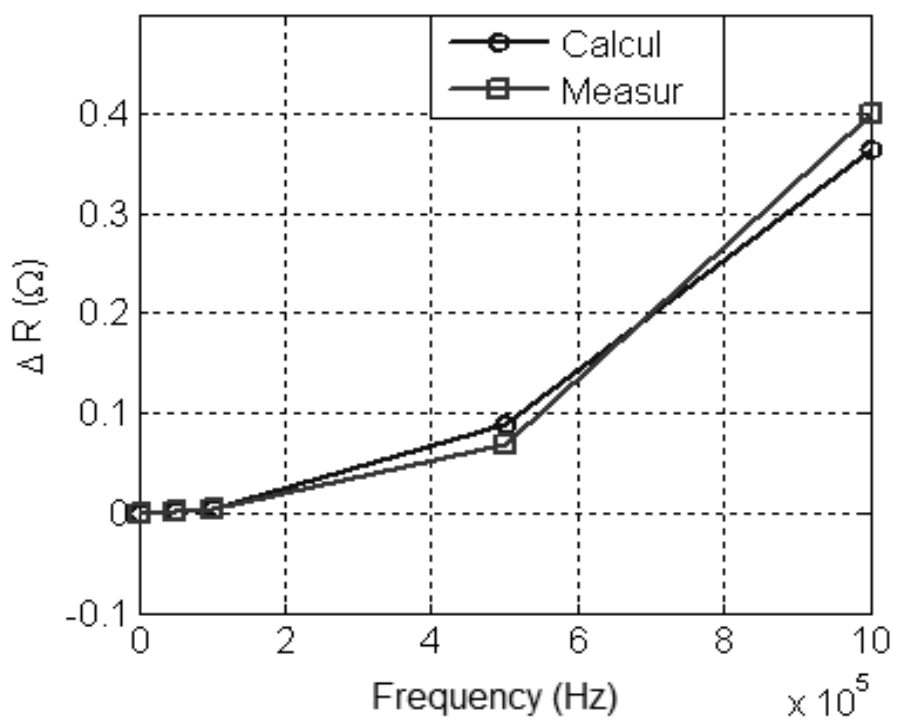

Fig. 7 
Tables

Table 1

\begin{tabular}{ll}
\hline Parameters & Values \\
\hline CFRP plate & \\
Thickness $(\delta)$ & $0.125 \mathrm{~mm}$ \\
Length $(\mathrm{L})$ & $150 \mathrm{~mm}$ \\
Width $(\mathrm{W})$ & $100 \mathrm{~mm}$ \\
\hline Ferrite torus & $9 \mathrm{~mm}$ \\
Inner radius $\left(R_{i}\right)$ & $14 \mathrm{~mm}$ \\
Outer radius $\left(R_{e}\right)$ & $28.5 \mathrm{~mm}$ \\
Width $(\tau)$ & $0.59 \mathrm{~mm}$ \\
Air-gap thickness $(\mathrm{e})$ & $252(\mathrm{at} 100 \mathrm{kHz})$ \\
Relative permeability $\left(\mu_{r}\right)$ & \\
\hline Coil & $2 \mathrm{~mm}$ \\
Inner radius $\left(\mathrm{r}_{i}\right)$ & $4 \mathrm{~mm}$ \\
Outer radius $\left(\mathrm{r}_{e}\right)$ & $1.5 \mathrm{~mm}$ \\
Height $(h)$ & $0.1 \mathrm{~mm}$ \\
Lift off $(l)$ & 120 \\
Number of turns &
\end{tabular}

Table 2

\begin{tabular}{|c|c|c|c|c|c|c|}
\hline$\theta^{\circ}$ & $\left|\Delta \mathbf{R}_{\theta}\right|(\Omega)$ & $a_{\theta}$ & $\alpha_{\theta}$ & $\boldsymbol{\beta}_{\theta}$ & $\sigma_{L}(\mathrm{~S} / \mathbf{m})$ & $\sigma_{T}(\mathbf{S} / \mathbf{m})$ \\
\hline $0^{\circ}$ & 135 & 0.3978 & 0.2328 & 0.7860 & \multirow{2}{*}{$5.64 \times 10^{3}$} & \multirow{2}{*}{129.2} \\
\hline $90^{\circ}$ & 60 & 0.3535 & 0.0642 & 0.9422 & & \\
\hline
\end{tabular}

